OPEN ACCESS

Edited by:

Chad A. Bousman,

University of Calgary,

Canada

Reviewed by:

Andrea Gaedigk,

Children's Mercy Hospital,

United States

Todd Skaar,

Indiana University Hospital,

United States

${ }^{*}$ Correspondence:

Jeffrey A. Shaman

jshaman@coriell.com

tThese authors have contributed equally to this work.

Specialty section:

This article was submitted to

Molecular Psychiatry,

a section of the journal

Frontiers in Psychiatry

Received: 22 March 2019

Accepted: 03 June 2019

Published: 20 June 2019

Citation:

Jarvis JP, Peter AP and Shaman JA

(2019) Consequences of CYP2D6

Copy-Number Variation for

Pharmacogenomics in Psychiatry.

Front. Psychiatry 10:432.

doi: 10.3389/fpsyt.2019.00432

\section{Consequences of CYP2D6 Copy-Number Variation for Pharmacogenomics in Psychiatry}

\author{
Joseph P. Jarvis ${ }^{\dagger}$, Arul Prakasam Peter ${ }^{\dagger}$ and Jeffrey A. Shaman* \\ Coriell Life Sciences, Philadelphia, PA, United States
}

Pharmacogenomics represents a potentially powerful enhancement to the current standard of care for psychiatric patients. However, a variety of biological and technical challenges must be addressed in order to provide adequate clinical decision support for personalized prescribing and dosing based on genomic data. This is particularly true in the case of CYP2D6, a key drug-metabolizing gene, which not only harbors multiple genetic variants known to affect enzyme function but also shows a broad range of copynumber and hybrid alleles in various patient populations. Here, we describe several challenges in the accurate measurement and interpretation of data from the CYP2D6 locus including the clinical consequences of increased copy number. We discuss best practices for overcoming these challenges and then explore various current and future applications of pharmacogenomic analysis of CYP2D6 in psychiatry.

Keywords: personalized medicine, precision medicine, gene deletion, gene duplications, pharmacogenomics, cytochrome P450 CYP2D6, psychiatry, copy-number variation

\section{INTRODUCTION}

\section{Genetic Variation, Drug Response, and CYP Genes}

The clinical application of genomic technologies to enhance prescribing and the customization of pharmaceutical treatment plans is broadly known as pharmacogenomics (PGx). The basic principles of PGx are 1) that genetic variation in key genes involved in the processing and transport of pharmacological agents and their metabolites may alter clinical outcomes in meaningful, patientspecific ways and 2) that similar genetic variation in pharmacological targets may impact an individual's sensitivity to the effects of particular drugs (1). Though a wide variety of genes have been identified as important players in PGx, the most clinically useful and best studied are members of the cytochrome P450 superfamily. This large group of $>50$ human genes shows broad similarity in DNA sequence, though members catalyze a variety of reactions. Of clinical importance, several enzymes participate in the phase I detoxification pathway including well-known PGx loci such as CYP2C9, CYP2C19, and CYP2D6 (2).

\section{Single-Nucleotide Polymorphism (SNP) Variation}

Over the last two decades, results from published studies in basic science research and clinically oriented journals strongly support the use of PGx in clinical practice $(3,4)$. Many detail the impact of specific variants, usually single-nucleotide polymorphisms (SNPs), in "CYP" genes on the resulting enzyme's function. These so-called spelling errors in the genome impart a range of physiological consequences including no change, a measured reduction in protein function, 
a complete obliteration of function, or even an increase in enzymatic activity. Sometimes, genetic variation involves small insertions or deletions of base pairs instead of the substitution of one base pair for another. This form of variation is known as an "INDEL," which is a portmanteau of "insertion" and "deletion." They are often grouped together with substitution mutations such as transitions from A to $G$ or transversions from $\mathrm{A}$ to $\mathrm{C}$ because they tend to have comparable and often deleterious effects on enzyme function. For most locations in the genome, two copies are inherited-one from the mother and one from the father-that together have the potential to influence patient physiology.

When a patient carries two decreased function alleles, their anticipated physiological state with respect to drug metabolism by that enzyme may be deemed "normal metabolizer," NM for some genes-including CYP2D6-as in the case of a recent CPIC genotype-to-phenotype translation standardization project (5). However, the literature also contains historical interpretations of this combination as "intermediate metabolizer," IM, or "poor metabolizer," PM, depending on the particular gene and the specific combination of alleles present (6). Clinically, the reduction or elimination of enzyme function may contribute to an altered response to drug therapy. Depending on the specific pharmaceutical substrate in question, IMs and PMs may fail to clear standard doses of medication or their bioactive metabolites at a normal rate, thus leading to an increased risk of side effects. Alternatively, IMs and PMs may be unable to activate prodrugs such that the patient may fail to fully benefit from the prescribed therapy.

When a patient carries two increased function alleles, or three or more functional gene copies at a given gene of interest, their anticipated physiological state is termed "ultrarapid metabolizer," UM. When one increased function allele is paired with one normal function allele, overall rates of metabolism are termed "ultrarapid metabolizer," or the similar "rapid metabolizer," RM. Clinically, increased metabolism of a drug delivered in its active form may require an increased dose or dividing a higher dose over multiple doses per day in order to achieve consistent therapeutic levels. This is due to the increased metabolism of the drug into its inactive, excretable form. For prodrugs, an alternative therapy or lowered dose may be advisable because a greater-than-usual amount of metabolized, circulating active compound is expected, which may lead to an increased risk of negative side effects (7-9).

\section{Copy-Number Variants (CNVs)}

Another type of genetic change with profound implications for PGx are copy number variants or "CNVs." In loci that show such variation, two or more copies of the same gene sequence may be inherited from a single parent or the gene may be deleted altogether. Thus, the total number of open reading frames available for the production of fully functional, impaired, or enhanced proteins (depending on the pattern of other variants present), may differ substantially from the expected value of two gene copies. In some cases, the total copy number may be zero or as high as 10 or more (10), which can present in a variety of potential combinations (e.g., five from each parent, six copies from one and four from another, and seven copies from one and three from the other). Clinically, this added dimension of genetic variation can greatly impact the expected physiology for a given set of observations, often introducing significant ambiguity into the process of interpreting patient-specific outcomes (see Table 1). For example, when three or more of the duplicated alleles show normal function based on their pattern of SNP and INDEL variants, a state of "ultrarapid metabolism" may occur-a greater amount of functional protein is expected via

TABLE 1 | Potential consequences of duplication for interpreting patient metabolizer status.

\begin{tabular}{|c|c|c|c|c|c|c|}
\hline \multicolumn{3}{|c|}{$\begin{array}{l}\text { Hypothetical metabolizer phenotypes involving non-duplicated } \\
\text { alleles }\end{array}$} & \multicolumn{4}{|c|}{ Consequences of duplication } \\
\hline Metabolizer status & Alleles and activity & $\begin{array}{l}\text { Anticipated } \\
\text { response }\end{array}$ & $\begin{array}{l}\text { Adding a } \\
\text { normal allele }\end{array}$ & $\begin{array}{l}\text { Adding a } \\
\text { decreased activity } \\
\text { allele }\end{array}$ & $\begin{array}{l}\text { Adding an increased } \\
\text { activity allele }\end{array}$ & $\begin{array}{l}\text { Adding a non- } \\
\text { functional allele }\end{array}$ \\
\hline Normal metabolizer (NM) & $\begin{array}{l}\text { Two normal activity } \\
\text { alleles, combination } \\
\text { of one increased } \\
\text { activity allele and one } \\
\text { decreased activity } \\
\text { allele }\end{array}$ & $\begin{array}{l}\text { Typical } \\
\text { metabolism }\end{array}$ & $\begin{array}{l}\text { Increased } \\
\text { metabolism }\end{array}$ & $\begin{array}{l}\text { Potentially increased } \\
\text { metabolism }\end{array}$ & Increased metabolism & Typical metabolism \\
\hline Intermediate metabolizer (IM) & $\begin{array}{l}\text { One normal activity } \\
\text { allele with one non- } \\
\text { functional activity } \\
\text { allele, two decreased } \\
\text { activity alleles }\end{array}$ & $\begin{array}{l}\text { Decreased } \\
\text { metabolism }\end{array}$ & $\begin{array}{l}\text { Typical or } \\
\text { decreased } \\
\text { metabolism }\end{array}$ & $\begin{array}{l}\text { Likely decreased } \\
\text { metabolism }\end{array}$ & Uncertain metabolism & $\begin{array}{l}\text { Decreased } \\
\text { metabolism }\end{array}$ \\
\hline Poor metabolizer (PM) & $\begin{array}{l}\text { Only non-functional } \\
\text { alleles detected }\end{array}$ & $\begin{array}{l}\text { Little or no } \\
\text { metabolism }\end{array}$ & $\begin{array}{l}\text { Decreased } \\
\text { metabolism }\end{array}$ & $\begin{array}{l}\text { Likely decreased } \\
\text { metabolism }\end{array}$ & $\begin{array}{l}\text { Likely decreased } \\
\text { metabolism }\end{array}$ & $\begin{array}{l}\text { Little or no } \\
\text { metabolism }\end{array}$ \\
\hline Ultrarapid metabolizer (UM) & $\begin{array}{l}\text { Two increased } \\
\text { activity alleles }\end{array}$ & $\begin{array}{l}\text { Increased } \\
\text { metabolism }\end{array}$ & $\begin{array}{l}\text { Increased } \\
\text { metabolism }\end{array}$ & Increased metabolism & Increased metabolism & Increased metabolism \\
\hline
\end{tabular}

(Left panel) Hypothetical metabolizer phenotypes for specific combinations of non-duplicated alleles with differing activity levels and expected patient physiology, (right panel) predicted changes in metabolizer status when a specific type of additional allele is present (CNV =3). Ambiguous or uncertain interpretations are shaded red. 
transcription/translation from the greater than two open reading frames. While they may seem to be a somewhat exotic form of variation, CNVs are actually quite common-roughly $12 \%$ of the human genome shows some degree of copy-number variation (11), and this includes key PGx genes including CYP2D6 (1215). In fact, one study showed that $12.6 \%$ of the general US population had copy-number variation in the CYP2D6 gene (16).

\section{Gene-Conversion Events}

Additionally, due to the high degree of sequence similarity among CYP genes, gene-conversion events involving various members including CYP2D6 have been observed. In a gene-conversion event, a portion of one DNA sequence is effectively pasted over the top of some portion of another's, creating a hybrid gene containing sequence from both loci. In the case of CYP2D6, a wide variety of such hybrid alleles between it and the nearby CYP2D7 pseudogene have been described (17-21). Clinically, as with many genomic changes, such rearrangements often result in decreased function or non-functional proteins (22).

\section{Patient Stratification}

Regardless of the type(s) of variation involved, many PGx studies include important insights into the way patients that share a particular metabolizer status differ in their response to the therapeutic substrate being studied. However, very few of these studies contain outright and immediately adoptable clinical insights. For example, according to the highly cited PGx knowledge base PharmGKB, only $\sim 7 \%$ of medications have associated genomic information that may be acted upon directly by a physician (1). Interestingly however, these compounds represent $\sim 18 \%$ of all prescriptions written in the US (1). Further, recent reports indicate a large proportion of individuals carry at least one PGx-actionable variant (23) with many bearing two or more. This suggests that the majority of the patient population shows at least some potential to encounter a drug for which PGx information is available. However, the ultimate clinical utility of patient genetic data often depends on assessment and interpretation of the complete combination of variants they possess rather than the presence of one or two variants. So while it is clear that genetic stratification of patients can be a valuable aid to medical practitioners, ordering commercially available PGx reports should be regarded as an enhancement of, rather than a replacement for, current standards of care.

\section{Potential Benefits of PGx to Psychiatry}

Clinically speaking, the field of psychiatry shows strong potential to disproportionately benefit from the adoption of PGx than do other specialties. Perhaps the most important reason is the relatively high rate of poor clinical outcomes for patients under standard care. For example, between $30 \%$ and $50 \%$ of psychiatric patients do not respond sufficiently to acute treatment no matter which medication is originally prescribed (24-32), and only $35 \%$ to $45 \%$ of patients with major depressive disorder return to premorbid levels of function after 6-8 weeks of treatment (24). Thus, there is clearly room for genomic data to inform current clinical practice.
Further, psychiatry is the second most commonly observed primary therapeutic area (20.8\%, see Figure 1A), after oncology (31.9\%), on the list of all U.S. Food and Drug Administration (FDA)-approved drugs with available PGx information (33) (summarized in Table 2). So, as with oncology, the research literature clearly contains the raw materials for building valuable clinical decision support for psychiatrists. Finally, of those roughly $20.8 \%$ of FDA-approved drugs with PGx information that is used in psychiatry, $69.2 \%$ are fully processed in some way by a single gene: CYP2D6 (see Figure 1B). This represents a substantial enrichment of CYP2D6 substrates in psychiatric drugs since, overall, CYP2D6 is known to impact the metabolism of $25 \%$ of all FDA-approved medications. These data suggest an excellent opportunity to focus on a high-value genomic region with great potential for improving patient outcomes. Thus, despite both biological and technical challenges to measuring and interpreting data from CYP2D6, the locus may hold the key to important improvements to the standard of care for psychiatric patients.

\section{CYP2D6: Structure, Observed Variation, and Nomenclature}

The CYP2D6 gene consists of nine exons and is found on the negative strand between 42,126,499 and 42,130,881 bp (GRCh38. p12) on chromosome 22q13.2. As shown in Figure 2, variation at CYP2D6 occurs in exons, introns, and both the upstream and downstream regions of the locus.

To date, a total of 198 separate variants of various types have been cataloged for CYP2D6 (18) (PharmVar version 3.4, Figure 2A). In order to arrive at a useful clinical interpretation, the subsets present in any given patient must be considered simultaneously. As such, the concept of the "haplotype"-commonly referred as "* alleles" (read as "star alleles") in CYP genes-and the related nomenclature standards (22) for CYP2D6 alleles are critical to understand. Here, "haplotype" refers to the precise combination of variants found on the physical strand of DNA inherited from a specific parent. The combination of the two haplotypes inherited from both parents is collectively known as the "diplotype," and it is generally the convention to list the lowest numbered haplotype first (34). Ultimately, it is important to remember that * alleles are CYP2D6 haplotypes that may involve multiple sites and types of variation.

Often, the same SNP may be found on multiple genetic backgrounds, which, based on the totality of variants present, show different activities (rsIDs in black in Figure 2). Further, many named/characterized collections of variants are defined by large numbers of genetic changes (Figure 3 and Supplemental Figures 1-4, see ${ }^{*} 35 B\left(C Y P 2 D 6^{*} 35.002\right)$, which contains 38 variants, and ${ }^{\star} 2 A\left(C Y P 2 D 6^{\star} 2.001\right)$, which bears 16$)$, some of which are shared by multiple named alleles. For example, the $\mathrm{C}>\mathrm{T}$ variant in exon 1 known as rs1065852 (22:42130692 in GRCh37, c.100C > T, p.Pro34Ser, P34S) is present in at least 36 distinct haplotypes (Figure 2). Thus, one important technical challenge for converting raw genetic data into an accurate determination of diplotype is to understand which variants were inherited together from each parent. 


\section{A Pharmacogenomic Information on Drug Labels by Therapeutic Class}

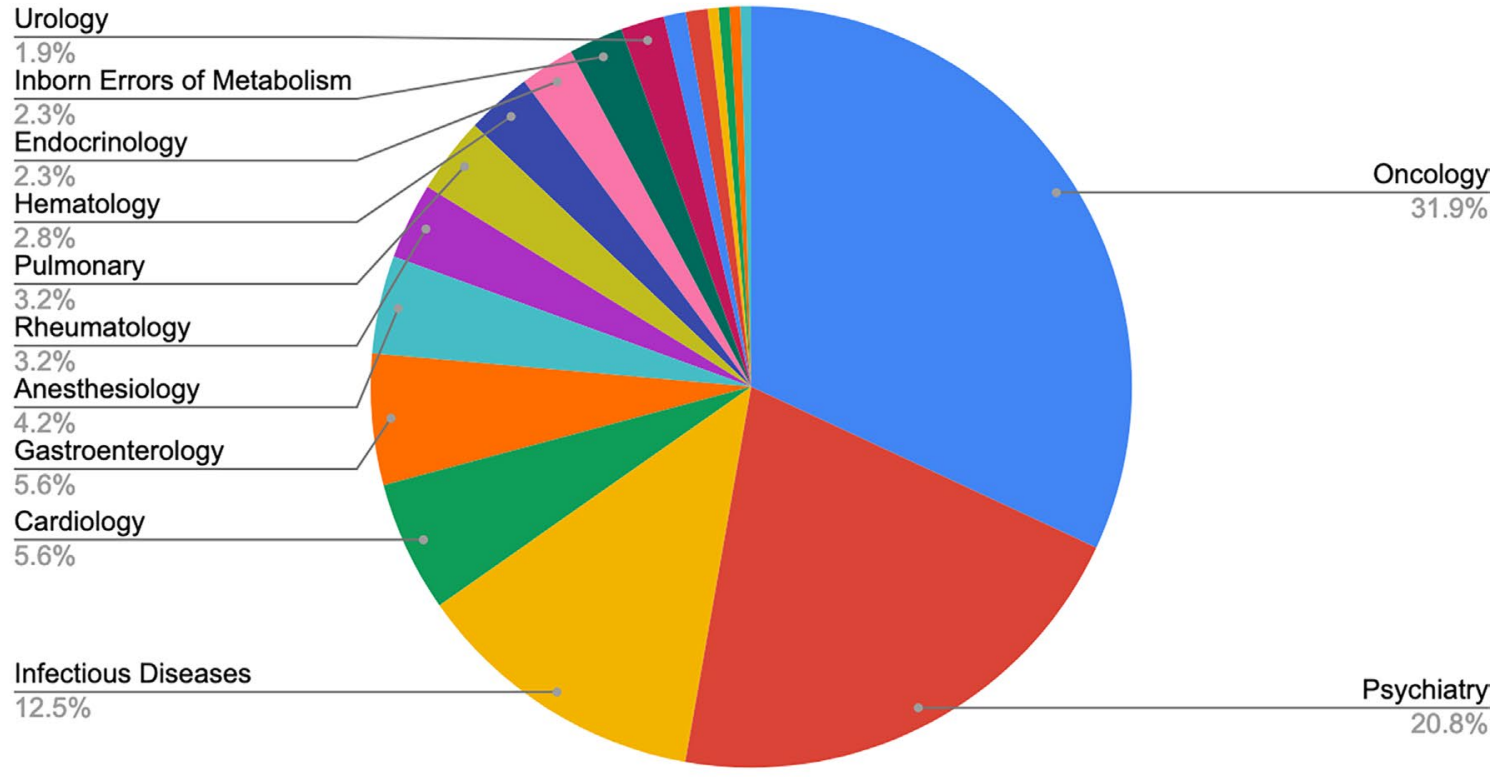

\section{B Genes that Influence Psychiatric Drugs}

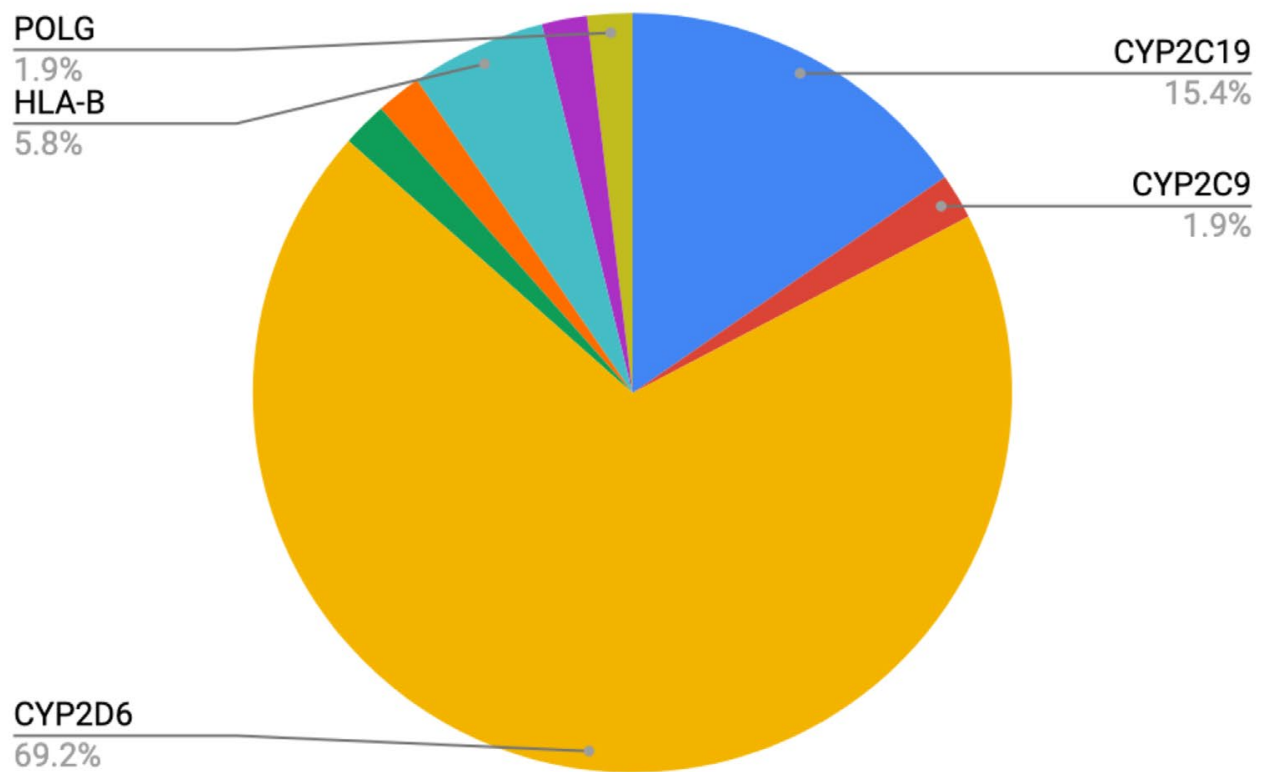

FIGURE 1 | Percentages of (A) FDA-approved drugs with available biomarker information in their labels by therapeutic class (total $N=215$ ) and (B) FDA-approved psychiatric drugs influenced by specific PGx genes of interest (total $N=45$, see also Table 2).

Once characterized, each unique haplotype that has been observed is assigned its own " "*) designation and is logged in various databases and public resources $(35,36)$. In the most simple cases, a single variant fully defines a haplotype. For example, the presence of a "C" at position rs5030867 is currently all that is needed to identify a ${ }^{\star} 7$ haplotype at CYP2D6. In more complicated cases, the simultaneous presence of many types of variation (e.g., SNPs, CNV, and gene-conversion events) defines the haplotypes carried by a given patient. In order to properly identify such complex * alleles, measurements at all-or nearly all—sites of variation are required. When no variation is observed at any tested site, the haplotype is assigned a designation of ${ }^{*} 1$ by default. 
TABLE 2 | The 45 drug products that are FDA-approved for use in neurology and psychiatry that contain pharmacogenomic information in their drug labels, and the gene/biomarker of interest for each. A total of 36 drug labels include actionable or informative pharmacogenomic information in labeling sections Dosage and Administration, Warnings and Precautions, Adverse Reactions, Drug Interactions, Clinical Pharmacology, and Use in Specific Populations (33).

\begin{tabular}{|c|c|}
\hline Drug & Biomarker \\
\hline Amitriptyline ${ }^{\dagger}$ & CYP2D6 \\
\hline Aripiprazole Rx,Pop,C.Ph. & CYP2D6 \\
\hline Aripiprazole Lauroxil Rx,Pop,C.Ph. & CYP2D6 \\
\hline Atomoxetine Rx,!t,ADR,DDI,C.Ph. & CYP2D6 \\
\hline Brexpiprazole Rx,Pop,C.Ph. & CYP2D6 \\
\hline Brivaracetam C.Ph. & CYP2C19 \\
\hline Carbamazepine !,BW,t,! & $H L A-A, H L A-B$ \\
\hline Cariprazine C.Ph. & CYP2D6 \\
\hline Citalopram Rx,!,C.Ph. & CYP2C19 \\
\hline Citalopram C.Ph. & CYP2D6 \\
\hline Clobazam Rx,Pop,C.Ph. & CYP2C19 \\
\hline Clomipramine $^{\dagger}$ & CYP2D6 \\
\hline Clozapine Rx,Pop,C.Ph. & CYP2D6 \\
\hline Desipramine $^{\dagger}$ & CYP2D6 \\
\hline Desvenlafaxine ${ }^{\text {C.Ph. }}$ & CYP2D6 \\
\hline Deutetrabenazine Rx,lt,Pop,C.Ph. & CYP2D6 \\
\hline Dextromethorphan and Quinidine !t,C.Ph. & CYP2D6 \\
\hline Diazepam C.Ph. & CYP2C19 \\
\hline Doxepin C.Ph., C.Ph. & CYP2D6, CYP2C19 \\
\hline Duloxetine DDI & CYP2D6 \\
\hline Escitalopram DDI, ADR & CYP2D6, CYP2C19 \\
\hline Eteplirsen I\&U,ADR,Pop,Cli & $D M D$ \\
\hline Fluoxetine ${ }^{t, C . P h}$. & CYP2D6 \\
\hline Fluvoxamine DDI & CYP2D6 \\
\hline Galantamine C.Ph. & CYP2D6 \\
\hline Iloperidone Rx,It,DDI,C.Ph. & CYP2D6 \\
\hline Imipramine $^{\dagger}$ & CYP2D6 \\
\hline Lacosamide $^{\text {C.Ph. }}$ & CYP2C19 \\
\hline Meclizine ${ }^{\text {C.Ph. }}$ & CYP2D6 \\
\hline Modafinil C.Ph. & CYP2D6 \\
\hline Nefazodone $^{\dagger}$ & CYP2D6 \\
\hline Nortriptyline ${ }^{\dagger}$ & CYP2D6 \\
\hline Oxcarbazepine!t & $H L A-B$ \\
\hline Paroxetine DDI & CYP2D6 \\
\hline Perphenazine ${ }^{\text {t,C.Ph. }}$ & CYP2D6 \\
\hline Phenytoin C.Ph., C.Ph.,! & CYP2C9, CYP2C19, HLA-B \\
\hline Pimozide Rx, $\uparrow$ & CYP2D6 \\
\hline Protriptyline $^{\dagger}$ & CYP2D6 \\
\hline Risperidone DDI,C.Ph. & CYP2D6 \\
\hline Tetrabenazine Rx,!t,Pop,C.Ph. & CYP2D6 \\
\hline Thioridazine $\mathrm{Cl}^{\prime, !+}$ & CYP2D6 \\
\hline Trimipramine ${ }^{\dagger}$ & CYP2D6 \\
\hline Valbenazine Rx,It,Pop,C.Ph. & CYP2D6 \\
\hline Valproic Acid BW,Cl,!t, Cl,!t & POLG, nonspecific \\
\hline Venlafaxine $^{\dagger}$ & CYP2D6 \\
\hline Vortioxetine Rx,C.Ph. & CYP2D6 \\
\hline
\end{tabular}

ADR, Adverse Reactions; BW, Boxed Warning; C.Ph., Clinical Pharmacology; $\mathrm{Cl}$, Contraindications; Rx, Dosage and Administration; DDI, Drug Interactions; t. Precautions; Pop, Use in Specific Populations; !, Warnings; ! t, Warnings and Precautions; I\&U, Indications and Usage; Cli, Clinical Studies.

When copy-number variation is observed for one or more of the haplotypes, the notation for the duplicated allele is included as " $x N$," with $N$ being the number of copies of the specific allele when it is possible to determine. For example, a CYP $2 D 6{ }^{\star} 1 \times 2 /{ }^{*} 2$ diplotype indicates that one allele carries a ${ }^{\star} 1$ gene duplication while the other allele carries one ${ }^{\star} 2$ gene copy.

\section{CYP2D6 Haplotyping, SNP Assays, and Clinical Interpretation: Considerations and Challenges}

There is an important technical challenge in resolving certain haplotype combinations involving heterozygous variants at multiple locations that does not occur when the observed variation is homozygous. Specifically, if two variants are observed at the same location (e.g., a test result for a specific variant is homozygous in the absence of a whole gene duplication or deletion event), one must have come from one parent and the other from the other parent. Thus, the pattern of inheritance is clear. However, if two variants are observed at different locations, it is unclear whether both variants came from one parent or one variant was inherited from each. The consequences of multi-variant genotypes are particularly complex when they involve changes that completely eliminate enzyme function. Patients showing these combinations have inherited either two non-functional copies of the gene or one normal copy, which is paired with a single non-functional copy carrying both variants. These two possibilities may have very different physiological interpretations depending on the drug involved and consequently different clinical implications. Real-world situations can be substantially more complex to resolve than this simple two-locus example, and novel alleles may also be observed in some patients, which can greatly complicate clinical reporting. Unambiguous ascertainment of the specific distribution of variants on each chromosome yields "phased" haplotypes, something very few of the current technologies are able to produce. Rather, phase is usually estimated using existing knowledge of haplotypes that are expected in the patient genepool and/or via mathematical algorithms.

At present, the CYP2D6 haplotypes that confer increased overall function do so via increased translation of mRNA to protein due to the presence of two or more gene sequences conferring normal function (CNV-variable haplotypes, see below and Figure 4). As such, they must be identified by an assay developed specifically for the purposes of their detection. A similar issue arises with gene conversion polymorphisms that produce hybrid alleles. Since assays for the various types of variation showing measurable consequences for overall CYP2D6 activity in patients are usually performed independently, uniting raw data from all sources into a coherent picture of clinical actionability can be quite challenging. Furthermore, since the effects of specific combinations show a range of physiological effects from non-functional CYP2D6 protein to an increased rate of CYP2D6 enzyme metabolism, clinical interpretation of even accurate genetic profiles can be difficult and are sometimes ambiguous.

It should also be noted that a large number of haplotypes show either unknown-combinations that are too rare or for which there is too little published data to effectively interpretor, uncertain function - that is, test results/research findings that are conflicting or inconclusive. Diplotypes involving haplotypes with unknown or uncertain functions are particularly difficult to interpret in clinically useful ways, though case studies involving them will be useful in resolving ambiguities. For example, a case study of a non-responding patient who carries a known haplotype with inconsistent evidence in other studies but who 


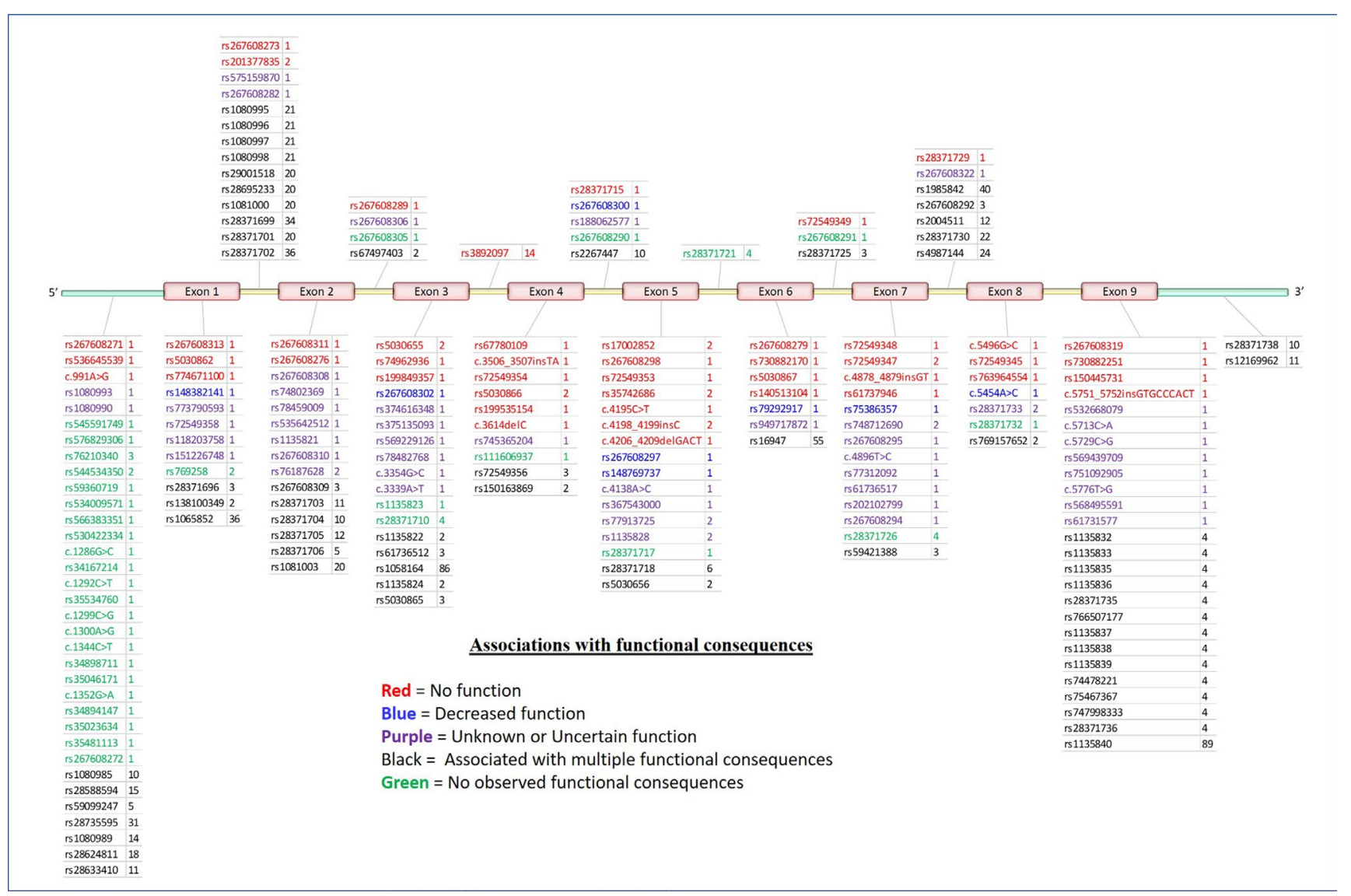

FIGURE 2 I 5' to $3^{\prime}$ structure of the CYP2D6 locus and placement of variants. Physical position of known pharmacogenomics (PGx) variants in CYP2D6 by rsID (total $N=198$ ). Colors indicate putative functional consequences: red $=$ no function, blue $=$ decreased function, purple $=$ unknown or uncertain function, black = single-nucleotide polymorphism (SNPs) with multiple associated functional consequences [e.g., rs1135840 can be found in alleles with normal function, decreased function, and even non-functional (e.g., ${ }^{\star} 35$, ${ }^{\star} 17$, and ${ }^{*} 4$, respectively)], green $=$ no observed functional consequences to date (normal). Numbers to the right of each rSID indicate the total number of haplotypes ( ${ }^{*}$ alleles) on which each variant is known to be found.

clearly benefits from a change in therapy would be a helpful observation and suggests potentially fruitful avenues of future research. These avenues may include in vivo phenotyping or pharmacokinetic studies of similar individuals that shed further light on the function of the allele in question.

When patient diplotypes include two haplotypes with clearly defined functions, they may be grouped into potentially substrate-specific metabolizer status groups including normal (NM, previously called extensive, EM), intermediate (IM), poor (PM), and ultrarapid (UM). Depending on a full understanding of clinical and basic science research, the expected physiological consequences of membership in each group may then be developed into clinical decision support.

\section{CYP2D6: CNV-Variable Haplotypes}

The CYP2D6 ${ }^{*} 5$ allele is a complete deletion of the gene sequence that can be inherited from one or both parents. Functionally, as no protein can be produced from the $\star_{5}$ allele, it imparts a complete elimination of CYP2D6 enzymatic function. Therefore, in the physiological interpretation of metabolizer status, it is generally treated in a similar way as other alleles lacking functionality and represents the extreme end of the functional continuum for all substrates. However, there are technical challenges that arise when ${ }^{*} 5$ alleles are present. For example, when ${ }^{\star} 5$ is paired with a ${ }^{*} 1$ allele (i.e., no SNP or INDEL variants are observed), technical limitations may cause the patient to appear to carry two normally functioning alleles $\left({ }^{*} 1 /{ }^{*} 1\right)$ until copy-number status is measured (37). Thus, in the absence of CNV data, accurate clinical interpretation of results may not be possible even for examples that appear to be relatively simple from a genetic perspective. Similar complications arise when ${ }^{*} 5$ is paired with alleles carrying various combinations of variants-the technical results will appear as though the patient is homozygous for all observed variants rather than a heterozygous together with a $* 5$ allele. This, in turn, has the potential to introduce considerable ambiguity in clinical interpretation.

Just as there can be wholesale deletions of a CYP2D6 allele, the chromosomal region where CYP2D6 is found can carry two or more copies of the CYP2D6 gene. Such duplications can involve gene units that are functional (e.g., ${ }^{\star} 1 x N$ and ${ }^{\star} 2 x N$ ) or nonfunctional (e.g., ${ }^{\star} 4 x N$ ) or those that show decreased function (e.g., ${ }^{\star} 41 x N$ ), leading to a variety of potential clinical consequences. 


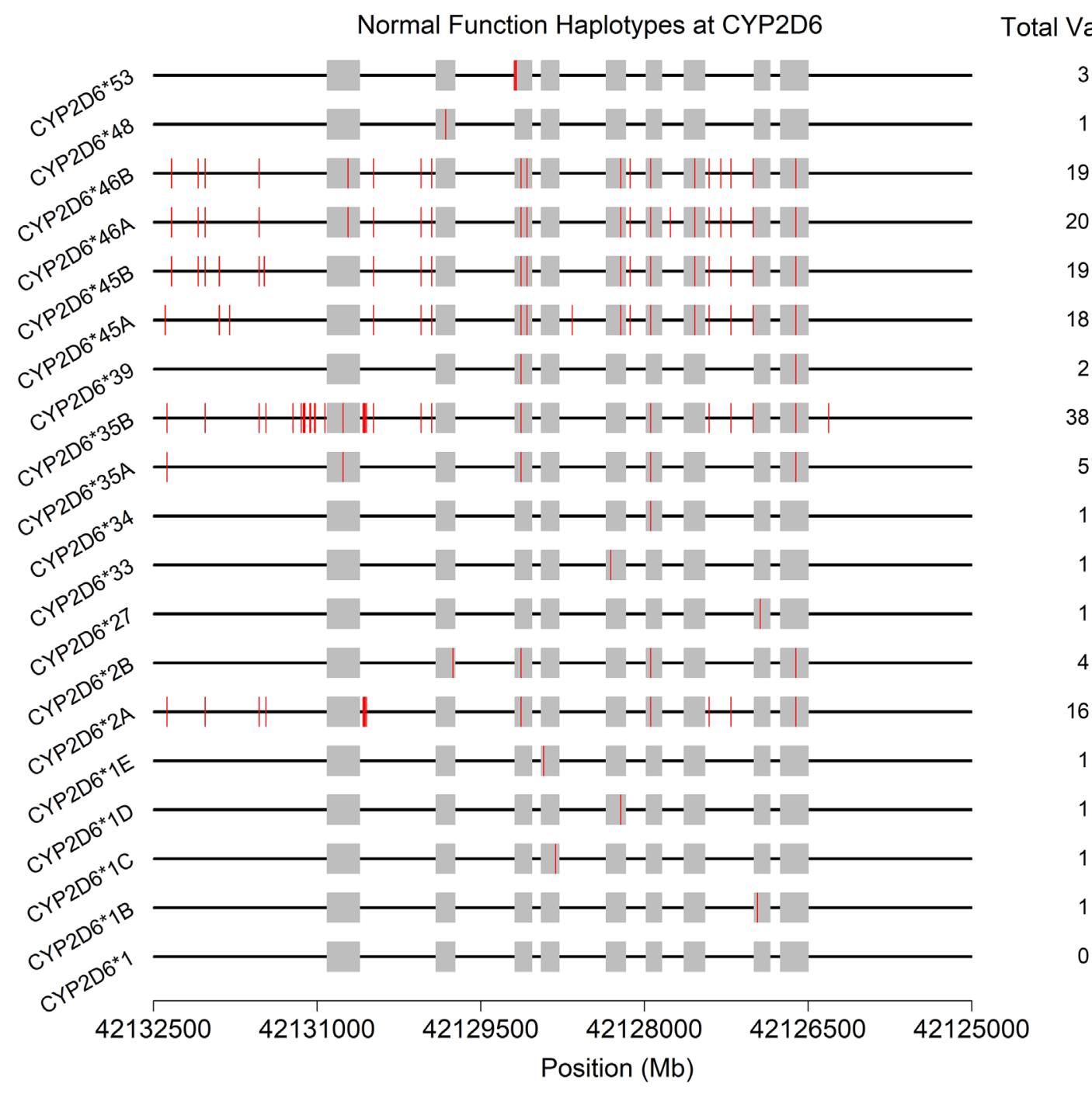

FIGURE 3 | Physical positions (red vertical lines) and total number of variants found on the 19 haplotypes (* alleles) predicted to produce normally functioning enzymes upon transcription/translation. See also Supplemental Figures 1-4. Gray boxes indicate the exons (genome build 37).

To date, 14 haplotypes bearing various combinations of SNPs and INDELs have been observed to be duplicated in one or more individual $(14,38)$ (Figure 4A). The most commonly observed duplicated alleles are ${ }^{\star} 1,{ }^{\star} 2$, and ${ }^{\star} 4$ (15). While other duplications appear more rarely, they do occur at appreciable frequencies in clinical populations and thus must be considered when resolving diplotype combinations and reporting their clinical consequences. It should be noted that, since current catalogs of human genomic variation are incomplete, and especially so for populations of non-European origins, other duplications involving known and as-yet-undocumented alleles likely exist somewhere in the human population. As databases and other genomic resources improve in their sampling of globally diverse populations over time, both the accuracy of diplotyping and the accuracy of the associated clinical decision support are expected to improve.

\section{CYP2D6 CNV Assays: Considerations and Challenges}

While the technical sensitivity of laboratory assays for CNVs at CYP2D6 can vary, some are capable of accurately discerning the total number present up to 5 and even 10 copies. However, many assays can only provide a $\mathrm{CNV}$ resolution of $>2$, and in the majority of cases, resolution becomes less certain at levels $>4$. Critically, and as noted above, the CNV and SNP/INDEL assays are often performed independently, and available databases/ catalogs of variation are incomplete. Thus, it can be difficult to determine which * allele should be assigned which copy-number. For example, for a patient assayed as CYP $2 D 6{ }^{\star} 1 /{ }^{\star} 2$ with a copynumber of 4 , a fully descriptive clinical report should present the results as “* $1 \times 3 /{ }^{\star} 2$, or ${ }^{\star} 1 \times 2 /{ }^{\star} 2 \times 2$, or ${ }^{\star} 1 /{ }^{\star} 2 \times 3$," because the haplotype of the duplicated gene was not determined with 


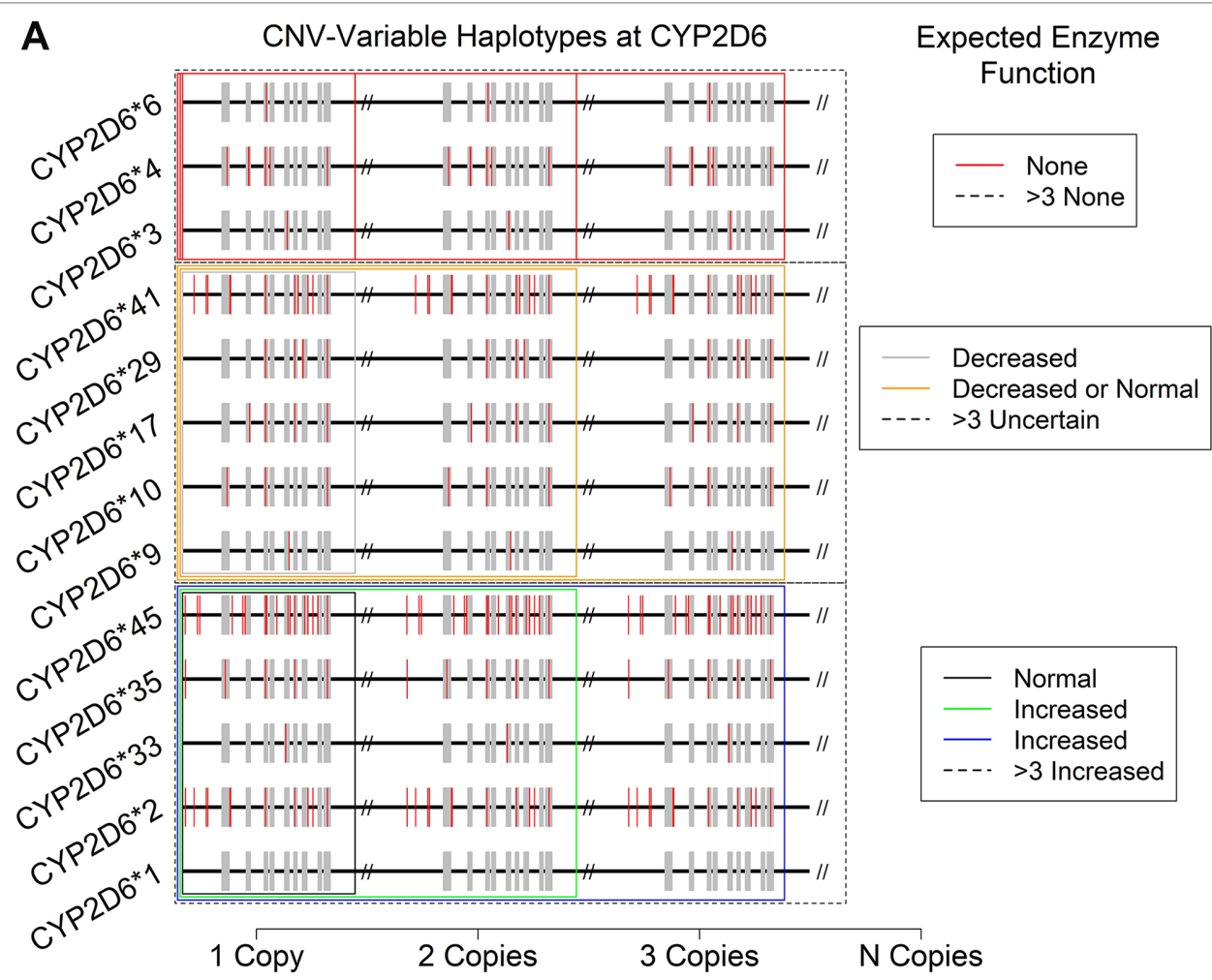

B

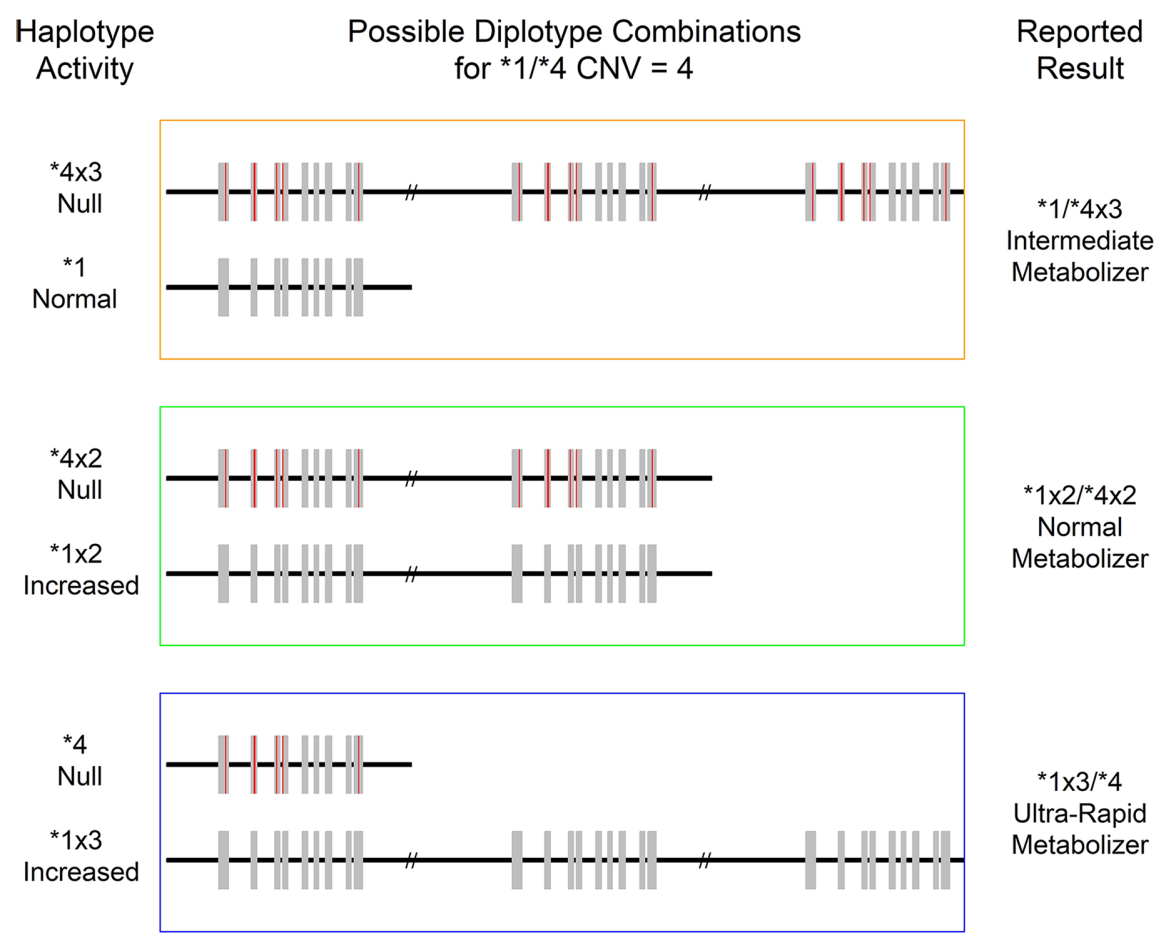

FIGURE 4 | Consequences of CYP2D6 gene duplication for (A) the activity level of 13 expected CNV-variable (duplicated) CYP2D6 alleles with known enzyme function. Not depicted: CYP2D6 *43, which is also known to show duplications with an uncertain phenotype. (B) Individual haplotype ( ${ }^{*}$ allele) activity and overall metabolizer status of the "* $1 / * 4, C N V=4$ " specific patient result. Note that a copy-number of four introduces ambiguity in the reported metabolizer status due to technical uncertainty regarding which specific allele is duplicated. 
certainty. Fortunately, in this case, the physiological interpretation of metabolizer status and thus the clinical consequences are identical for all three possibilities. Specifically, since both ${ }^{\star} 1$ and ${ }^{*} 2$ show normal function, each potential result yields the same ultrarapid metabolizer status designation (Figure 4A, bottom boxes: black, green, and blue). Likewise, a CYP $2 D 6^{*} 4{ }^{\star}{ }^{\star} 6$ patient with any copy-number value may be interpreted as a PM since no matter how many copies of either allele are present, all protein produced is expected to be non-functional for the specific substrate of interest (Figure 4A, top red boxes).

The situation becomes increasingly complex when duplicated alleles with different functional characteristics are present. For example, in a $C Y P 2 D 6{ }^{*} 1 /{ }^{\star} 4$ patient with $C N V=4$ (Figure $4 \mathrm{~B}$ ), there are again three distinct possibilities for their diplotype: ${ }^{*} 1 \times 3 /{ }^{*} 4,{ }^{*} 1 \times 2 /{ }^{*} 4 \times 2$, and ${ }^{*} 1 /{ }^{*} 4 \times 3$. However, in this case, each is associated with a distinct metabolizer status (UM, NM, and IM, respectively), and so each may have a different clinical interpretation (e.g., increased dose, standard dose, or decreased dose of a drug delivered in its active form). Such ambiguous results should be interpreted with caution and in concert with the specific patient's medical and drug response history (if available). Previous adverse drug reactions and past medication efficacy may or may not shed light on the actual diplotype and metabolizer status present. This combination of genetic testing and traditional clinical approaches to treatment likely represents a best-case scenario for certain genetically complex results.

\section{CYP2D6 Ultrarapid Metabolizers in Clinical Practice}

The measurement of total copy-number at the CYP2D6 locus is particularly crucial for PGx in clinical psychiatry. Currently, the only known way for CYP2D6 metabolism to be increased is via duplication of one or more of the CNV-variable * alleles with normal or decreased function. Further, the most useful PGxbased clinical decision support for many of the drugs used in psychiatry can be provided for ultrarapid metabolizers, which, by definition, must carry one or more duplicated allele.

While there are no overarching guidelines for the use of metabolizer status in clinical practice, strong evidence-based research and outcomes data support their utility in many contexts. For instance, the US FDA's Center for Drug Evaluation and Research allows for and approves the addition of metabolic status and dosing impacts and warnings directly to drug labels. Similarly, independent pharmacogenomic consortia have included actionable PGx information in guidelines. For example, the Dutch Pharmacogenetics Working Group (DPWG) has 47 guidelines (39), and the Clinical Pharmacogenetics Implementation Consortium (CPIC) has 19 peer-reviewed and published guidelines on 40 gene-drug pairs (40). These guidelines commonly highlight ultrarapid metabolism at CYP2D6 as important for the care of patients.

Indeed, it has long been known that additional functional copies of CYP2D6 impact the pharmacokinetics of various substrates including nortriptyline and debrisoquine $(38,41)$. It was shown that elimination rates of nortriptyline were fivefold higher than those of PMs for subjects carrying just a single additional copy of CYP2D6, that is, three total copies (38). When 13 functional copies were present, the rate was 17 -fold higher than for subjects with no active CYP2D6 enzyme. Further, it was subsequently shown that quinidine inhibition of debrisoquine metabolism in individuals carrying 3, 4, or 13 normal copies of CYP2D6 could be used to potentially alter clinical outcomes. These data together suggest that advance knowledge of a patient's metabolizer status at CYP2D6 via genetic testing could be invaluable in avoiding issues such as treatment resistance and/ or toxicity (42).

Similar information has the potential to greatly inform the choice of therapy and dosage in multiple contexts since CYP2D6 processes several clinically valuable anxiolytics, antidepressants, and antipsychotics (see Table 2). For example, a meta-analysis reports that the dosage of $\sim 50 \%$ of commonly used antipsychotics is dependent on CYP2D6 genotype (24). In addition, extraordinarily high clearance rates of the antidepressant trimipramine have been observed in three carriers of duplications at CYP2D6 taken from a group of healthy subjects. Ultimately, the authors suggest that a dose of up to $200 \%$ of average may be required for such individuals to attain similar concentrations as normal metabolizers (43). Further, since CYP2D6 is responsible for the hydroxylation of trimipramine and not its methylation (44), the effects of CYP2D6 UM status on the active metabolite desmethyltrimipramine must be considered as well since it is also metabolized by CYP2D6. Thus, the potential difference in clinical effects may be even larger than considering trimipramine alone (43).

The same study explored the effects of duplication at CYP2D6 on the tricyclic antidepressant doxepin in healthy subjects. The authors observed that ultrarapid metabolizers showed levels of the active metabolite $N$-desmethyldoxepin at $40 \%$ of those seen in normal metabolizers, and considering both this active daughter compound and levels of doxepin itself, NMs showed levels two-fold higher than did UMs. Similarly, interpretable trends are seen for the tricyclic antidepressants imipramine and desipramine $(10,45)$.

In a retrospective study of non-responsiveness to antidepressants metabolized by CYP2D6, a complete absence of UMs was observed in the subset of patients $(N=28)$ experiencing adverse drug events (ADEs), while eight were identified as PMs. The authors conclude that this enrichment is four-fold higher than expected by chance alone. Conversely, in a subset of 16 non-responders without ADE, no UMs and only one PM were observed (10). While admittedly a small sample size, these trends strongly support the role of CYP2D6 duplications in important clinical outcome measures. Finally, in a study that identified 81 non-responders to antidepressants metabolized by CYP2D6, 10\% (eight subjects) carried duplications. The authors point out that this is a substantial enrichment over the $0.8-1.0 \%$ incidence expected for Nordic Caucasians and that the worst week scores of the Hamilton Depression Rating Scale were greater in those carrying duplicated alleles than in those who did not $(10,46)$.

The clearance of the $S(+)$ form of mirtazapine shows a similar pattern across metabolizer groups and has been observed to be 1.6-fold higher in CYP2D6 UMs than in NMs (47). Since the $R(-)$ form is not metabolized by CYP2D6 $(48,49)$ but does appear to 
be responsible for cardiovascular ADEs, UMs may be at higher risk for both therapeutic failure and side effects when prescribed high doses of mirtazapine $(10,47)$. Additionally, therapeutic failure due to the effects of increased metabolism by CYP2D6 can increase suicidal behavior in depression patients (50-52). Moreover, UMs have been found to have an elevated risk of high scores on one of the Hamilton Depression Rating Scales that measures suicidality among unipolar or bipolar depressive inpatients (53). UMs also may show low plasma concentrations of fluoxetine or amitriptyline in monotherapy than do PMs, IMs, and NMs if starting concentrations are at the low end of the range when treating major depressive disorder (54).

In one of the largest long-term patient-blinded randomized controlled trials [Genomics Used to Improve DEpression Decisions (GUIDED)], consisting of 1,167 outpatients diagnosed with major depressive disorder (MDD) and patient- or clinicianreported inadequate response to at least one antidepressant, it was found that treating with pharmacogenetic testing-guided therapy, when compared with treatment as usual, at week 8 showed statistically significant improvements in response $(26.0 \%$ versus $19.9 \%$ ) and remission (15.3\% versus $10.1 \%)$. These results further support the potential role of pharmacogenomic testing in the guided treatment of difficult-to-treat psychiatric patients and the improvement of response and remission rates (55).

Ultrarapid metabolism by CYP2D6 has also been suggested to interact with other genetic factors to influence treatment response in certain patient groups. For example, a recent paper suggests that venlafaxine-XR remission is more common in patients with major depressive disorder who 1) failed to respond to citalopram/ escitalopram, 2) had CYP2D6 ultrarapid metabolism, and 3) carried certain allele combinations at SLC6A4 and SLC6A2 (56). This potentially greater level of genetic resolution for clinical decision support suggests that finer and finer levels of specificity for specific patient groups may be possible in the future. In some cases, this may involve not only interactions attributable to metabolism of compounds by multiple genes at a particular stage of processing but also the action of the same gene at different stages of detoxification.

\section{DISCUSSION}

\section{Biology, Technology, Interpretations, and Clinical Decision Support}

The CYP2D6 locus shows a highly complex pattern of genetic variants that are inherited in a multitude of combinations. The effect of any given combination of variants on the translated protein can also vary considerably. In turn, clinical outcomes measured in patients carrying similarly functioning alleles also show a degree of variability, but also enough statistical consistency to show great promise for adding new insights to patient care and the enhancement of standard practice. In order to reap these benefits, however, the technical challenges associated with accurately capturing and interpreting raw, laboratory-derived data must be overcome by those who endeavor to provide clinical decision support based upon it. These include 1) accurately producing data for each SNP/INDEL, gene conversion, and copy-number variant, 2) arranging them into likely haplotypes, 3) inferring the metabolizer status that each combination is likely to impart, and 4) accurately connecting each status to the very latest clinical safety and efficacy information in the ever-evolving landscape of the primary literature. The final step, the burden of the health care provider, is of course integrating this information into treatment plans in ways that benefit patients in clinical scenarios.

Accurately assaying CNVs at the CYP2D6 locus is particularly critical to ensure maximal clinical benefits of testing. Without this key piece of information, very little confidence can be ascribed to results in many cases. Unfortunately, while FDAapproved methods for assaying variation at CYP2D6 (e.g., AmpliChip CYP450 from Roche and xTAG CYP2D6 kit from Luminex) take CNVs into account, some laboratory-developed tests (LDTs) do not (57). Indeed, a somewhat alarming recent publication examining PGx reports noted that nearly a third of those laboratories surveyed appear to have failed to incorporate CNVs into CYP2D6 testing (58). Furthermore, since methods to measure SNPs and INDELs are usually performed independently from techniques to measure CNVs, nearly all tests are limited in their ability to merge these multiple sources of data into definitive haplotypes (i.e., by unequivocally assigning all variants to specific copies of the gene) $(15,59)$. As we have also seen, the presence of an allelic deletion (the CYP2D6 ${ }^{*} 5$ allele) produces complications from both a technical and reporting perspective. However, while current laboratory methods are likely imperfect, they nonetheless produce clinically useful insights overall with strong potential to support the creation of patient-specific treatment plans. In fact, it has been estimated that the costs to treat "extreme" metabolizers at CYP2D6 (either PMs or UMs), compared with NMs, can be as high as $\$ 4,000$ to $\$ 6,000$ more per year $(60,61)$, and so advance knowledge of patient metabolizer status may help both reduce costs and increase the quality of care.

\section{Best Practices}

Ultimately, there are several recommendations for best practices for the use of PGx in psychiatry. First, as with any clinical assay, it is important to choose clinical pharmacogenomic tests that are performed in a Clinical Laboratory Improvement Amendments (CLIA) accredited laboratory. These are laboratories that perform human, clinical testing rather than genetic testing for research purposes. They are required to adhere to an established set of rules and regulations and are audited on a regular basis. Second, choose an assay that measures a reasonable subset of CYP2D6 SNPs, INDELs, and CNVs. Assays performed to detect SNP/INDEL variation should be capable of capturing the key * alleles that are appropriate to the relevant patient population. With respect to CNVs, assays should be capable of discerning quantitative differences in total allele number between 0 and 5 copies at a minimum. Third, for reporting and interpretation purposes, the effects of all measured variation on enzyme function should be considered simultaneously as haplotypes rather than one variant at a time. This means that CYP450 results should be reported as diplotypes (pairs of * alleles) rather than the presence and/or absence of specific individual variants 
alone. Further, all possible results from ambiguous combinations should be reported when they arise (e.g., ${ }^{\star} 1 \times 3 /{ }^{*} 2$, or ${ }^{\star} 1 \times 2 /{ }^{\star} 2 \times 2$, or $\left.{ }^{\star} 1 /{ }^{*} 2 \times 3\right)$.

Finally, when reviewing results, findings should be placed in their proper clinical context and appreciated for the clinical utility that they may or may not represent. For example, results such as "CYP2D6 ${ }^{*} 1 /{ }^{*} 1, \mathrm{CNV}=2$ " (i.e., no observed variation by the assay in question) are not simply "non results" but rather indicate that standard precautions and procedures are appropriate with the acknowledgement that unmeasured variants may be present, a clinically useful insight. Further, reports showing ambiguous findings-in terms of the so-called diplotypes, metabolizer status, or the potential clinical impact of the observed variants in a given patient-may not provide clear clinical actions but may be useful in the context of patient history and other clinical factors in the determination of medication therapy management. For example, genetics may indicate that a patient may be either a UM or an NM at CYP2D6, but adverse reactions in their medical record may strongly suggest that one status is more likely than the other. Such cases represent important case studies that should be considered for publication. Other unique clinical scenarios also exist and complicate the interpretation of genetic results. For example, liver transplant recipients are expected to have complex medication processing profiles that resemble the metabolizer status of the donor rather than status indicated by the patient's own DNA. Clearly, such factors are beyond the scope of laboratory testing based on blood, saliva, or cheek swaps, and thus the responsibility falls to the provider to integrate all relevant information into an overall picture of patient care.

\section{Clinical Significance of Population Effects and CYP2D $6 *^{*} 1 /{ }^{*} 1$}

The frequencies of certain variants, including some key duplicated alleles, show non-trivial levels of variation across global populations (62). There are multiple clinically relevant effects of this observation. The first and most important is that the ${ }^{\star} 1$ designation is currently most appropriate for populations of European origin and may be seriously misleading for individuals with recent ancestry from other continental populations. Current genomic databases of all types, including those widely used for PGx assay development, severely under-represent global genomic diversity. Thus, many SNPs, INDELs, and CNVs with potentially important effects on enzyme function and clinical outcomes are simply not cataloged and so do not appear in laboratory-developed CYP2D6 assays. As such, until knowledge bases and other resources are more complete, a certain degree of caution should be exercised when interpreting ${ }^{\star} 1 /{ }^{\star} 1$ results.

Further, allele frequency differences across continental populations are likely to affect a wide variety of loci genomewide. Since Phase I detoxification is a complicated process, involving multiple enzymes, that is integrated with many other biological systems, some amount of variation in the effects of PGx loci is expected. For example, if processing by CYP2D6 is generally the rate-limiting step in the metabolism of a particular drug, but another locus is responsible for the processing of a bio-active metabolite, changes in allele frequencies at the second locus may well alter the clinically observable effects of the measured CYP2D6 variants. Thus, any given patient of average European lineage may show a larger or smaller effect size attributable to CYP2D6 for a given combination of variants than is expected based on potentially measurable variation at other loci contributing to overall patient physiology.

\section{The Future of PGx Testing and Clinical Guidance}

Despite acknowledged limitations, PGx testing is clearly clinically useful now. This is especially true in psychiatric care. As an enhancement to current practice and an important source of insight into patient physiology and expected drug response, PGx can help ensure maximally effective and minimally risky treatment plans, improve patient outcomes, and contribute to much-needed efficiency in health care spending. Interestingly, these benefits are only expected to increase given 1) the large volume of quality research being published annually, 2) the maintenance and curation of critical knowledge bases focused on aggregating key findings, 3) the development of multiple commercial products ensuring both academic and industry engagement in the field, 4) the growing adoption of PGx in clinical practice, and 5) the ultimate emergence of clear practice guidelines. The broader application of PGx and of prescription decision support tools (63) in routine practice is especially important as it will provide invaluable opportunities to define and refine fruitful hypotheses and targeted research initiatives that directly connect research efforts to endpoints and outcomes of clear clinical importance. More specifically, the evolving utility and expansion in the scope of PGx will be facilitated over the nearterm by developments in three key areas: 1) surmounting current technological limitations, 2) the need for clear outcomes data for each medication/diplotype combination, and 3) leveraging PGx research and associated databases to study the genetic influence on endogenous compound metabolism and xenobiotics more generally.

As explained above, there is currently no single technology that can accurately, efficiently, and simultaneously assay all critical types of genetic variation and unequivocally connect them to the specific DNA molecule inherited from a specific parent. This includes most strategies for whole genome sequencing, which also cannot reliably produce this sort of "fully phased" genomic data. However, there are promising techniques in development that are beyond the scope of this review to explore in detail such as long-read, single-molecule sequencing methods (64) that potentially offer this level of genomic resolution. Further, the ability to unambiguously determine the location of every variant across every copy of the gene present in a specific patient would have a profound impact on our understanding of the clinical impact of CNVvariable haplotypes. Once the challenges associated with these approaches are addressed, it will be possible to examine the full picture of genomic variation at key loci such as CYP2D6 in a patient-by-patient fashion and thus more accurately place 
them in clinically relevant groupings according to expected enzyme function. Coupled with improved physiology testing approaches to understanding the detoxification process, this enhanced level of detail will undoubtedly uncover specific subsets of patients who will disproportionately benefit or who are at disproportionate risk during the application of particular therapeutic strategies.

However, this appropriate phenotypic grouping of patients (e.g., by metabolizer status) also relies on further refinements in our understanding of the clinical impact of specific, potentially rare combinations of variants. As such, it will also be critical to the future development of PGx reporting that key outcomes data continue to be pursued in multiple populations of interest on a diplotype-by-diplotype basis. The enhancement to our wider understanding of the utility of genomic variation provided by such efforts will undoubtedly allow the transition of PGx interpretations from "informative" on current FDA drug labels to "actionable." It will also increase confidence in reporting for populations that are understudied at present and likely allow more detailed dosing information (such as those available for aripiprazole) to be available for larger number of pharmaceuticals. Ideally, this work would be performed in large samples of human patients, though this may not be fully feasible due to practical limitations including the very large number of potential confounding variables. Alternatively, in exploring and establishing the "true" gradations of decreased function between non-functional and normal, it may be advantageous to augment human studies with work in cell culture or model organisms in order to achieve sufficient sample sizes and statistical support for physiologically relevant findings.

Finally, the expansion in scope of PGx from the genetics of processing/transport and clinical effects of pharmaceutically marketed compounds into other areas of biochemistry and pharmacokinetics is also clearly on the horizon. For example, CYP2D6 has been identified as a potential metabolizer of endogenous neuroactive substrates (65), suggesting future applications of accurate data from CYP2D6 in psychiatric care involving internal homeostatic processes/physiology in the "normal" range and natural disease progression. Further, the genetic architecture responsible for processing commercially produced compounds is the very same that handles xenobiotic metabolism more generally. As such, the re-deployment of genome-wide PGx data in service of understanding the consequences of unintentional environmental exposures to chemicals such as perfluoroalkyl and polyfluoroalkyl substances (PFAS) and many other likely neurotoxins is likely to emerge as an important contributor to the future health and wellness of the general population.

\section{CONCLUSIONS}

The use of PGx as an enhancement to the current standard of care for psychiatric patients shows great potential to guide therapy and improve outcomes in a wide variety of clinical contexts. However, it should not be viewed as a panacea. Important limitations, both technical and biological, must be kept in mind, and PGx information should be carefully integrated with other patient-specific data in the development of customized treatment plans. Some of these limitations will continue to produce ambiguous results for some patients for the foreseeable future at loci like CYP2D6, especially when $\mathrm{CNVs}$ are present. However, many ambiguous results still provide useful and actionable information if they are fully explained and understood. Likewise, ${ }^{*} 1 /{ }^{\star} 1$ can also provide useful clinical guidance in supporting a standard course of treatment. However, they should also be considered with caution depending on the scope of the variants tested in a given report (i.e., more are usually better) and the population of origin of some patients (e.g., we should remember that knowledge bases are currently incomplete for those of non-European ancestry). Thus, follow-up testing with expanded assays, re-testing at some time in the future, or investing in continuously updated clinical interpretive reports may be useful in certain circumstances.

It is also important to maintain an awareness of developments in PGX as they occur in the coming years. Technological advancements are expected to enhance the utility of genomic data in the clinic, and the ever-expanding databases of clinical outcomes are likely to refine and expand the clinical decision support that is possible to deliver. Ultimately, PGx is a valuable tool in any clinician's toolkit, and its reasonable use in refining patient-specific treatment plans has the potential to greatly improve the health and well-being of many psychiatric patients.

\section{AUTHOR CONTRIBUTIONS}

All authors contributed to research, manuscript writing, and revisions and have read and approved the submitted version.

\section{FUNDING}

This work was supported by Coriell Life Sciences.

\section{ACKNOWLEDGMENTS}

We thank Paul R. Shaman, DDS, MS, and the reviewers whose suggestions helped improve and clarify this manuscript.

\section{SUPPLEMENTARY MATERIAL}

The Supplementary Material for this article can be found online at: https://www.frontiersin.org/articles/10.3389/fpsyt.2019.00432/ full\#supplementary-material 


\section{REFERENCES}

1. Relling MV, Evans WE. Pharmacogenomics in the clinic. Nature (2015) 526:343-50. doi: 10.1038/nature15817

2. Iyanagi T. Molecular mechanism of phase I and phase II drug-metabolizing enzymes: implications for detoxification. Int Rev Cytol (2007) 260:35-112. doi: 10.1016/S0074-7696(06)60002-8

3. Ventola CL. Pharmacogenomics in clinical practice: reality and expectations. P T (2011) 36:412-50.

4. Johnson JA. Pharmacogenetics in clinical practice: how far have we come and where are we going? Pharmacogenomics (2013) 14:835-43. doi: 10.2217/ pgs. 13.52

5. Clinical Pharmacogenetics Implementation Consortium (CPIC). CYP2D6 Genotype to Phenotype Standardization Project. (2019). Accessed May 9, 2019.

6. Wu AH. Drug metabolizing enzyme activities versus genetic variances for drug of clinical pharmacogenomic relevance. Clin Proteomics (2011) 8:12. doi: 10.1186/1559-0275-8-12

7. Ahmed S, Zhou Z, Zhou J, Chen SQ. Pharmacogenomics of drug metabolizing enzymes and transporters: relevance to precision medicine. Genomics Proteomics Bioinformatics (2016) 14:298-313. doi: 10.1016/j. gpb.2016.03.008

8. Roden DM, Wilke RA, Kroemer HK, Stein CM. Pharmacogenomics: the genetics of variable drug responses. Circulation (2011) 123:1661-70. doi: 10.1161/CIRCULATIONAHA.109.914820

9. Weinshilboum R. Inheritance and drug response. N Engl J Med (2003) 348:529-37. doi: 10.1056/NEJMra020021

10. Johansson I, Ingelman-Sundberg M. CNVs of human genes and their implication in pharmacogenetics. Cytogenet Genome Res (2008) 123:195204. doi: 10.1159/000184709

11. Yang Y, Botton MR, Scott ER, Scott SA. Sequencing the CYP2D6 gene: from variant allele discovery to clinical pharmacogenetic testing. Pharmacogenomics (2017) 18:673-85. doi: 10.2217/pgs-2017-0033

12. He Y, Hoskins JM, McLeod HL. Copy number variants in pharmacogenetic genes. Trends Mol Med (2011) 17:244-51. doi: 10.1016/j.molmed. 2011.01.007

13. Del Tredici AL, Malhotra A, Dedek M, Espin F, Roach D, Zhu GD, et al. Frequency of CYP2D6 alleles including structural variants in the United States. Front Pharmacol (2018) 9:305. doi: 10.3389/fphar.2018.00305

14. Gaedigk A, Ndjountche L, Divakaran K, Dianne Bradford L, Zineh I, Oberlander TF, et al. Cytochrome P4502D6 (CYP2D6) gene locus heterogeneity: characterization of gene duplication events. Clin Pharmacol Ther (2007) 81:242-51. doi: 10.1038/sj.clpt.6100033

15. Gaedigk A. Complexities of CYP2D6 gene analysis and interpretation. Int Rev Psychiatry (2013) 25:534-53. doi: 10.3109/09540261.2013.825581

16. Beoris M, Amos Wilson J, Garces JA, Lukowiak AA. CYP2D6 copy number distribution in the US population. Pharmacogenet Genomics (2016) 26:96-9. doi: 10.1097/FPC.0000000000000188

17. Gaedigk A, Bradford LD, Alander SW, Leeder JS. CYP2D6*36 gene arrangements within the cyp2d6 locus: association of CYP2D6*36 with poor metabolizer status. Drug Metab Dispos (2006) 34:563-9. doi: 10.1124/ dmd.105.008292

18. Pharmacogene Variation Consortium (PharmVar). Version 3.4.

19. Gaedigk A, Jaime LK, Bertino JS, Jr., Berard A, Pratt VM, Bradfordand LD, et al. Identification of novel CYP2D7-2D6 hybrids: non-functional and functional variants. Front Pharmacol (2010) 1:121. doi: 10.3389/fphar. 2010.00121

20. Sim SC, Daly AK, Gaedigk A. CYP2D6 update: revised nomenclature for CYP2D7/2D6 hybrid genes. Pharmacogenet Genomics (2012) 22:692-4. doi: 10.1097/FPC.0b013e3283546d3c

21. Gaedigk A, Fuhr U, Johnson C, Berard LA, Bradford D, Leeder JS. CYP2D7-2D6 hybrid tandems: identification of novel CYP2D6 duplication arrangements and implications for phenotype prediction. Pharmacogenomics (2010) 11:43-53. doi: 10.2217/pgs.09.133

22. Gaedigk A, Ingelman-Sundberg M, Miller NA, Leeder JS, Whirl-Carrillo M, Klein TE, et al. The Pharmacogene Variation (PharmVar) Consortium: incorporation of the human cytochrome P450 (CYP) allele nomenclature database. Clin Pharmacol Ther (2018) 103:399-401. doi: 10.1002/cpt.910
23. Mostafa S, Kirkpatrick CMJ, Byron K, Sheffield L. An analysis of allele, genotype and phenotype frequencies, actionable pharmacogenomic (PGx) variants and phenoconversion in 5408 Australian patients genotyped for CYP2D6, CYP2C19, CYP2C9 and VKORC1 genes. J Neural Transm (Vienna) (2019) 126:5-18. doi: 10.1007/s00702-018-1922-0

24. Kirchheiner J, Nickchen K, Bauer M, Wong ML, Licinio J, Roots I, et al. Pharmacogenetics of antidepressants and antipsychotics: the contribution of allelic variations to the phenotype of drug response. Mol Psychiatry (2004) 9:442-73. doi: 10.1038/sj.mp.4001494

25. Topic E, Stefanovic M, Ivanisevic AM, Blazinic F, Culav J, Skocilic Z. CYP2D6 genotyping in patients on psychoactive drug therapy. Clin Chem Lab Med (2000) 38:921-7. doi: 10.1515/CCLM.2000.135

26. Murphy GM, Jr., Kremer C, Rodrigues HE, Schatzberg AF. Pharmacogenetics of antidepressant medication intolerance. Am J Psychiatry (2003) 160:1830-5. doi: 10.1176/appi.ajp.160.10.1830

27. Malhotra AK, Murphy GM, Jr., Kennedy JL. Pharmacogenetics of psychotropic drug response. Am J Psychiatry (2004) 161:780-96. doi: 10.1176/appi.ajp.161.5.780

28. Corponi F, Fabbri C, Serretti A. Pharmacogenetics in psychiatry. $A d v$ Pharmacol (2018) 83:297-331. doi: 10.1016/bs.apha.2018.03.003

29. Foley KF, Quigley DI. Pharmacogenomic potential of psychiatric medications and CYP2D6. MLO Med Lab Obs (2010) 42:32-4.

30. Walden LM, Brandl EJ, Tiwari AK, Cheema S, Freeman N, Braganza N, et al. Genetic testing for CYP2D6 and CYP2C19 suggests improved outcome for antidepressant and antipsychotic medication. Psychiatry Res (2018). doi: 10.1016/j.psychres.2018.02.055

31. Rosenblat JD, Lee Y, McIntyre RS. The effect of pharmacogenomic testing on response and remission rates in the acute treatment of major depressive disorder: a meta-analysis. J Affect Disord (2018) 241:484-91. doi: 10.1016/j. jad.2018.08.056

32. Bousman CA, Arandjelovic K, Mancuso SG, Eyre HA, Dunlop BW. Pharmacogenetic tests and depressive symptom remission: a meta-analysis of randomized controlled trials. Pharmacogenomics (2019) 20:37-47. doi: 10.2217/pgs-2018-0142

33. U.S. Food and Drug Administration. Table of pharmacogenomic biomarkers in drug labeling. (2018). August 3.

34. Daly AK, Brockmoller J, Broly F, Eichelbaum M, Evans WE, Gonzalez FJ, et al. Nomenclature for human CYP2D6 alleles. Pharmacogenetics (1996) 6:193-201. doi: 10.1097/00008571-199606000-00001

35. Robarge JD, Li L, Desta Z, Nguyen A, Flockhart DA. The star-allele nomenclature: retooling for translational genomics. Clin Pharmacol Ther (2007) 82:244-8. doi: 10.1038/sj.clpt.6100284

36. Kalman LV, Agundez J, Appell ML, Black JL, Bell GC, Boukouvala S, et al. Pharmacogenetic allele nomenclature: international workgroup recommendations for test result reporting. Clin Pharmacol Ther (2016) 99:172-85. doi: 10.1002/cpt.280

37. Kim J, Lee SY, Lee KA. Copy number variation and gene rearrangements in CYP2D6 genotyping using multiplex ligation-dependent probe amplification in Koreans. Pharmacogenomics (2012) 13:963-73. doi: 10.2217/pgs.12.58

38. Dalen P, Dahl ML, Bernal Ruiz ML, Nordin J, Bertilsson L. 10-Hydroxylation of nortriptyline in white persons with $0,1,2,3$, and 13 functional CYP2D6 genes. Clin Pharmacol Ther (1998) 63:444-52. doi: 10.1016/ S0009-9236(98)90040-6

39. Bank PCD, Caudle KE, Swen JJ, Gammal RS, Whirl-Carrillo M, Klein TE, et al. Comparison of the Guidelines of the Clinical Pharmacogenetics Implementation Consortium and the Dutch Pharmacogenetics Working Group. Clin Pharmacol Ther (2018) 103:599-618. doi: 10.1002/cpt.762

40. Relling MV, Klein TE. CPIC: Clinical Pharmacogenetics Implementation Consortium of the Pharmacogenomics Research Network. Clin Pharmacol Ther (2011) 89:464-7. doi: 10.1038/clpt.2010.279

41. Dalen P, Dahl ML, Eichelbaum M, Bertilsson L, Wilkinson GR. Disposition of debrisoquine in Caucasians with different CYP2D6-genotypes including those with multiple genes. Pharmacogenetics (1999) 9:697-706. doi: 10.1097/01213011-199912000-00004

42. Dalen P, Dahl M, Andersson K, Bertilsson L. Inhibition of debrisoquine hydroxylation with quinidine in subjects with three or more functional CYP2D6 genes. Br J Clin Pharmacol (2000) 49:180-4. doi: 10.1046/j.13652125.2000.00120.x 
43. Kirchheiner J, Bertilsson L, Bruus H, Wolff A, Roots I, Bauer M. Individualized medicine-implementation of pharmacogenetic diagnostics in antidepressant drug treatment of major depressive disorders. Pharmacopsychiatry (2003) 36 Suppl 3: S235-43. doi: 10.1055/s-2003-45136

44. Bolaji OO, Coutts RT, Baker GB. Metabolism of trimipramine in vitro by human CYP2D6 isozyme. Res Commun Chem Pathol Pharmacol (1993) 82:111-20.

45. Schenk PW, van Fessem MA, Verploegh-Van Rij S, Mathot RA, van Gelder T, Vulto AG, et al. Association of graded allele-specific changes in CYP2D6 function with imipramine dose requirement in a large group of depressed patients. Mol Psychiatry (2008) 13:597-605. doi: 10.1038/sj.mp.4002057

46. Kawanishi C, Lundgren S, Agren H, Bertilsson L. Increased incidence of CYP2D6 gene duplication in patients with persistent mood disorders: ultrarapid metabolism of antidepressants as a cause of nonresponse. a pilot study. Eur J Clin Pharmacol (2004) 59:803-7. doi: 10.1007/s00228-003-0701-4

47. Brockmoller J, Meineke I, Kirchheiner J. Pharmacokinetics of mirtazapine: enantioselective effects of the CYP2D6 ultra rapid metabolizer genotype and correlation with adverse effects. Clin Pharmacol Ther (2007) 81:699-707. doi: 10.1038/sj.clpt.6100116

48. Timmer CJ, Sitsen JM, Delbressine LP. Clinical pharmacokinetics of mirtazapine. Clin Pharmacokinet (2000) 38:461-74. doi: 10.2165/ 00003088-200038060-00001

49. Dodd S, Boulton DW, Burrows GD, De Vane CL, Norman TR. In vitro metabolism of mirtazapine enantiomers by human cytochrome P450 enzymes. Hum Psychopharmacol (2001) 16:541-44. doi: 10.1002/hup.328

50. Zackrisson AL, Lindblom B, Ahlner J. High frequency of occurrence of CYP2D6 gene duplication/multiduplication indicating ultrarapid metabolism among suicide cases. Clin Pharmacol Ther (2010) 88:354-9. doi: 10.1038/ clpt.2009.216

51. Penas-Lledo EM, Dorado P, Aguera Z, Gratacos M, Estivill X, FernandezAranda F, et al. High risk of lifetime history of suicide attempts among CYP2D6 ultrarapid metabolizers with eating disorders. Mol Psychiatry (2011) 16:691-2. doi: 10.1038/mp.2011.5

52. Penas-Lledo EM, Blasco-Fontecilla H, Dorado P, Vaquero-Lorenzo C, Baca-Garcia E, Llerena A. CYP2D6 and the severity of suicide attempts. Pharmacogenomics (2012) 13:179-84. doi: 10.2217/pgs.11.146

53. Stingl JC, Viviani R. CYP2D6 in the brain: impact on suicidality. Clin Pharmacol Ther (2011) 89:352-3. doi: 10.1038/clpt.2010.239

54. Penas-Lledo EM, Trejo HD, Dorado P, Ortega A, Jung H, Alonso E, et al. CYP2D6 ultrarapid metabolism and early dropout from fluoxetine or amitriptyline monotherapy treatment in major depressive patients. $\mathrm{Mol}$ Psychiatry (2013) 18:8-9. doi: 10.1038/mp.2012.91

55. Greden JF, Parikh SV, Rothschild AJ, Thase ME, Dunlop BW, DeBattista C, et al. Impact of pharmacogenomics on clinical outcomes in major depressive disorder in the guided trial: a large, patient- and rater-blinded, randomized, controlled study. J Psychiatr Res (2019) 111:59-67. doi: 10.1016/j. jpsychires.2019.01.003
56. Ahmed AT, Biernacka JM, Jenkins G, Rush AJ, Shinozaki G, Veldic M, et al. Pharmacokinetic-pharmacodynamic interaction associated with venlafaxine-XR remission in patients with major depressive disorder with history of citalopram/escitalopram treatment failure. J Affect Disord (2018) 246:62-68. doi: 10.1016/j.jad.2018.12.021

57. Willyard C. Copy number variations' effect on drug response still overlooked. Nat Med (2015) 21:206. doi: 10.1038/nm0315-206

58. Bousman CA, Jaksa P, Pantelis C. Systematic evaluation of commercial pharmacogenetic testing in psychiatry: a focus on CYP2D6 and CYP2C19 allele coverage and results reporting. Pharmacogenet Genomics (2017) 27:387-93. doi: 10.1097/FPC.0000000000000303

59. Langaee T, Hamadeh I, Chapman AB, Gums JG, Johnson JA. A novel simple method for determining CYP2D6 gene copy number and identifying allele(s) with duplication/multiplication. PLoS One (2015) 10:e0113808. doi: 10.1371/journal.pone.0113808

60. Gardner KR, Brennan FX, Scott R, Lombard J. The potential utility of pharmacogenetic testing in psychiatry. Psychiatry J (2014) 2014:730956. doi: $10.1155 / 2014 / 730956$

61. Chou WH, Yan FX, de Leon J, Barnhill J, Rogers T, Cronin M, et al. Extension of a pilot study: impact from the cytochrome P450 2D6 polymorphism on outcome and costs associated with severe mental illness. J Clin Psychopharmacol (2000) 20:246-51. doi: 10.1097/00004714-200004000-00019

62. Gaedigk A, Sangkuhl K, Whirl-Carrillo M, Klein T, Leeder JS. Prediction of CYP2D6 phenotype from genotype across world populations. Genet Med (2017) 19:69-76. doi: 10.1038/gim.2016.80

63. Chang DD, Eyreeuro HA, Abbott R, Coudreaut M, Baune BT, Shaman JA, et al. Pharmacogenetic guidelines and decision support tools for depression treatment: application to late-life. Pharmacogenomics (2018) 19:1269-84. doi: $10.2217 /$ pgs-2018-0099

64. Qiao W, Yang Y, Sebra R, Mendiratta G, Gaedigk A, Desnick RJ, et al. Long-read single molecule real-time full gene sequencing of cytochrome P450-2D6. Hum Mutat (2016) 37:315-23. doi: 10.1002/humu.22936

65. Penas-Lledo EM, Llerena A. CYP2D6 variation, behaviour and psychopathology: implications for pharmacogenomics-guided clinical trials. Br J Clin Pharmacol (2014) 77:673-83. doi: 10.1111/bcp.12227

Conflict of Interest Statement: Authors JJ, AP, and JS were employed by company Coriell Life Sciences. JJ and JS have equity interest in Coriell Life Sciences.

Copyright (c) 2019 Jarvis, Peter and Shaman. This is an open-access article distributed under the terms of the Creative Commons Attribution License (CC BY). The use, distribution or reproduction in other forums is permitted, provided the original author(s) and the copyright owner(s) are credited and that the original publication in this journal is cited, in accordance with accepted academic practice. No use, distribution or reproduction is permitted which does not comply with these terms. 